\title{
Anomalous Anti-proton to Negative Pion Ratio as Revealed by Jet Quenching at RHIC
}

\author{
Ivan Vitev* and Miklos Gyulassy*† \\ * Department of Physics, Columbia University, 538 W. 120-th Street, New York, NY 10027, USA \\ $\dagger$ Collegium Budapest, Szentharomsag u.2 H-1014 Budapest, Hungary
}

\begin{abstract}
We study the apparent discrepancy between the standard PQCD predictions for the meson and baryon ratios and multiplicities at moderate high $p_{\mathrm{T}}>2 \mathrm{GeV}$ and recent experimental measurements in $A u+A u$ collisions at $\sqrt{s}_{N N}=130 \mathrm{GeV}$ at the Relativistic Heavy Ion Collider (RHIC). We show that the differences, most pronounced in central collisions, can be explained by a strong non-perturbative baryon Junction component, which dominates the currently accessible experimental $p_{\mathrm{T}}$ window and the non-abelian energy loss of fast partons propagating through hot and dense medium. The recently introduced two component hybrid model, which combines a quenched jet PQCD calculation in the Gyulassy-Levai-Vitev (GLV) formalism and a phenomenological "soft" part, is further elaborated to take into account the full 3D expansion in the pre-hadronization phase and include particle flavor dependent "soft" inverse slopes as suggested by the baryon Junction picture. We show that such approach can resolve what seems to be a factor of $\simeq 2$ difference in the moderate high $p_{\mathrm{T}}$ suppression of $\pi^{0}$ and $h^{-}$as recently reported by the PHENIX collaboration. The observed quenching of the high $p_{\mathrm{T}}$ particle spectra and the large $\bar{p} / \pi^{-}$and $p / \pi^{+}$ratios as a function of $p_{\mathrm{T}}$ are found to be consistent with a creation of a deconfined phase and non-abelian energy loss of fast partons in a plasma of initial gluon rapidity density $d N^{g} / d y \sim 1000$
\end{abstract}

\section{INTRODUCTION}

The preliminary data from the first $A u+A u$ run of the Relativistic Heavy Ion Collider (RHIC) at $\sqrt{s}_{N N}=130 \mathrm{GeV}$ was reported by the experimental collaborations (BRAHMS, PHENIX, PHOBOS and STAR) at the Quark Matter 2001 [1] conference. It suggests the observation of qualitatively new phenomena in comparison to the CERN Super Proton Synchrotron (SPS) $P b+P b$ program results at $\sqrt{s}_{N N}=17.4 \mathrm{GeV}$. First, the moderate high $p_{\mathrm{T}}$ spectra of neutral pions in the $10 \%$ central collisions were reported by PHENIX [2] to be suppressed by a factor $2-4$ relative to the PQCD predictions scaled by nuclear geometry $\left(T_{A B}(\mathbf{b})\right.$, the Glauber profile density per unit area at an impact parameter $\mathbf{b}$ ). Second, the analysis of semi-peripheral collisions by STAR [3] shows that the elliptic flow $v_{2}\left(p_{\mathrm{T}}\right)$ is compatible with the monotonically growing hydrodynamic predictions for small transverse momenta, but saturates at $\sim 0.15$ above $p_{\mathrm{T}}>2 \mathrm{GeV}$. Third, studies of the charged particle rapidity density as a function of centrality by PHOBOS and PHENIX [4] revealed the expected (but previously not observed) $T_{A B}(\mathbf{b}) \sim N_{\text {part }}^{4 / 3}$ mini-jet component [5]. Finally, in the $p_{\mathrm{T}}=1-3.5 \mathrm{GeV}$ window where both $\pi^{0}$ and $h^{-}$have been measured, the $\pi^{0}$ are quenched by a factor of 2 more than the negative hadrons.

While the data are preliminary, the analysis of Ref. [6] suggests that these first results from RHIC may be interpreted as evidence for the production of a dense gluon plasma in central and semi-central reactions. The key diagnostic tool for probing the properties of the medium in that analysis is jet quenching [7]. Estimates based on high $p_{\mathrm{T}}$ suppression of negative hadrons indicate that the initial gluon density may have reached $\rho_{g}(\tau \sim 0.2 \mathrm{fm} / \mathrm{c}) \sim 100 \rho_{0}$, where $\rho_{0}=0.15 \mathrm{fm}^{-3}$ is the nuclear saturation density. At RHIC 
energies, $\sqrt{s}_{N N}=100-200 \mathrm{GeV}$, the initial flux of high $p_{\mathrm{T}}$ jets becomes sufficiently large and the produced gluon plasma is sufficiently dense and long lived to make jet quenching finally observable. In contrast, at SPS energies the semi-hard $\pi^{0}$ spectrum reported by the WA98 collaboration [8] was found to be actually enhanced by a factor of 2 at $p_{\mathrm{T}} \sim 2-4 \mathrm{GeV}$ due to the Cronin effect [9]. We emphasize the importance of studying $p+A$ systems to resolve the strength of the Cronin versus nuclear shadowing effects on the high $p_{\mathrm{T}}$ yields.

An unexpected result at RHIC reported by PHENIX [10] was that the flavor composition of high $p_{\mathrm{T}}>2$ $\mathrm{GeV}$ negative and positive $p_{\mathrm{T}}$ differential hadron yields may actually be dominated by anti-protons and protons respectively. Such behavior has not been observed so far in $p p$ or $A A$ collisions. In the conventional QCD multi-particle production phenomenology high $p_{\mathrm{T}}$ hadrons arise from the fragmentation of colored partons, $(q, g)$, whose spectra are computable via PQCD. If hot and dense matter is created in $A A$ reactions, those partons naturally lose energy through elastic and radiative multi-particle final state interactions. The quenching of high $p_{\mathrm{T}}$ partons is thus expected to result in the quenching of all their hadronic fragments including $\bar{p}$ and $p$.

To resolve the new high $p_{\mathrm{T}}$ baryon puzzle at RHIC we propose that the non-perturbative baryon Junctions component [11] of hadronic spectra provides a natural mechanism that could account for the anomalously large baryon/meson ratios. This component competes with the semi-hard PQCD processes even in $p p$ and $p A$ reactions but has only now been exposed in central $A u+A u$ at RHIC, because the usual perturbative QCD pion and kaon component is strongly suppressed due to jet quenching. The Junction mechanism is further supported by the observation that in central collisions at midrapdity $\bar{p} / p \approx 0.65$ and $d N^{B} / d y \sim$ $d N^{p} / d y-d N^{\bar{p}} / d y \sim 15$ [12], indicating a very significant baryon number transport (stopping) five units of rapidity from the fragmentation regions.

\section{HARD AND SOFT HADRONIC COMPONENTS}

The PQCD approach expresses the differential hadron cross section in $p+p \rightarrow h+X$ through a convolution of the measured structure functions $f\left(x_{\alpha}, Q_{\alpha}^{2}\right)_{\alpha / p}$ for the interacting partons $(\alpha=a, b)$, with the parameterized fragmentation function $D_{h / c}\left(z, Q_{c}^{2}\right)$ for the leading scattered parton $c$ into a hadron of flavor $h$ and the elementary parton-parton cross sections $d \sigma^{(a b \rightarrow c d)} / d \hat{t}$. Next to leading order corrections are accounted for by the constant $K \sim 2$. Other degrees of freedom for PQCD fits to data include the choice of the renormalization scale $Q^{2}$ and the parton broadening due to initial state radiation, which can be included through a normalized $k_{\mathrm{T}}$-smearing distribution. A simple functional form is the Gaussian smearing $f\left(k_{\mathrm{T}}\right)=e^{-k_{\mathrm{T}}^{2} /\left\langle k_{\mathrm{T}}^{2}\right\rangle} / \pi\left\langle k_{\mathrm{T}}^{2}\right\rangle$, where $\left\langle k_{\mathrm{T}}^{2}\right\rangle \simeq 0.6-1.0 \mathrm{GeV}^{2}$ in $p p, \bar{p} p$ collisions. In $A A$ reactions one expects larger initial parton broadening due to $k_{\mathrm{T}}$ kicks. In the absence of a medium, the invariant hadron inclusive cross section is given by

$$
\begin{aligned}
E_{h} \frac{d \sigma_{h}^{p p}}{d^{3} p}= & K \sum_{a b c d} \int d z_{c} d x_{a} d x_{b} \int d^{2} \mathbf{k}_{\mathrm{T} a} d^{2} \mathbf{k}_{\mathrm{T} b} f\left(\mathbf{k}_{\mathrm{T} a}\right) f\left(\mathbf{k}_{\mathrm{T} b}\right) f_{a / p}\left(x_{a}, Q_{a}^{2}\right) f_{b / p}\left(x_{b}, Q_{b}^{2}\right) \\
& D_{h / c}\left(z_{c}, Q_{c}^{2}\right) \frac{\hat{s}}{\pi z_{c}^{2}} \frac{d \sigma^{(a b \rightarrow c d)}}{d \hat{t}} \delta(\hat{s}+\hat{u}+\hat{t}),
\end{aligned}
$$

where $x_{a}, x_{b}$ are the initial momentum fractions carried by the interacting partons, $z_{c}=p_{h} / p_{c}$ is the momentum fraction carried by the observed hadron.

In the case of jet production inside a hot and dense medium, the fast parton loses a fraction of its energy due to gluon radiation induced by final state interactions. The full solution for the gluon radiative double differential distributions for the case of finite jet energies and few collisions was found in [7] in a form easily integrated numerically (to $\Delta E$ ) taking into account both the kinematic bounds and the running coupling $\alpha_{s}\left(Q^{2}\right)$. 

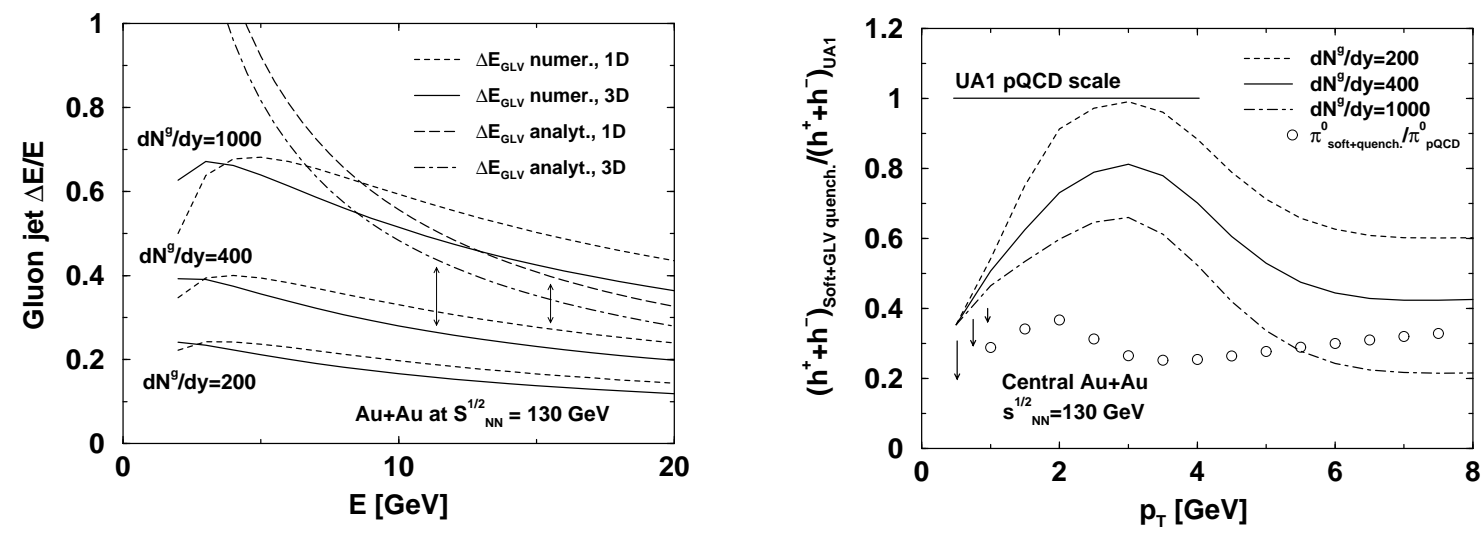

FIGURE 1. In Fig. 1a the gluon jet fractional energy loss in the GLV formalism is shown for three initial gluon rapidity densities $d N^{g} / d y=200,400,1000$ for both $1 \mathrm{D}$ and 3D expansion. The asymptotic analytic approximation ( shown here for $d N^{g} / d y=400$ ) is inapplicable at RHIC. Fig. 1b shows the ratio of charged particles computed in the soft+GLV quenched model relative to the conventional PQCD that accounts for the UA1 data. Also shown is the greater quenching predicted for $\pi^{0}$ with $d N^{g} / d y=400$.

We have also studied the effects of a strong transverse flow in addition to the boost-invariant longitudinal Bjorken expansion [13] and for the azimuthally averaged energy loss and found the simple result:

$$
\Delta E_{3 \mathrm{D}}^{(1)} \approx \frac{\delta}{1+\delta v_{\perp}} \Delta E_{1 \mathrm{D}}^{(1)}, \quad z_{\max }=\delta R
$$

where $v_{\perp}$ is the average transverse expansion velocity and $z_{\max }=\delta R$ is the duration of the energy loss phase $\left(\delta<1 /\left(1-v_{\perp}\right)\right)$. While the azimuthal asymmetry of $\Delta E$ is sensitive to the details of the expansion, the mean is not. For $v_{\perp}=0.6$ and $\beta=1.5 \Delta E_{3 \mathrm{D}}^{(1)} \simeq 80 \% \Delta E_{1 \mathrm{D}}^{(1)}$. Gluon jet mean fractional energy loss for three typical plasma densities is shown in Fig. 1a. Comparison to the asymptotic result signifies the importance of finite kinematics. Non-abelian energy loss affects hadronization through rescaling the hadron momentum fractions $z_{c}$ and modifying the fragmentation functions in Eq. (11) [6]. We note that because of the GLV energy loss $\Delta E / E \propto \rho_{g}$ is very weakly energy dependent (see Fig. 1a), the effect of energy loss fluctuations, can be absorbed as a gluon density renormalization by a factor $\sim 2-2.5$ [14].

The PQCD fragmentation picture gives adequate description of particle multiplicities in $p p$ and $\bar{p} p$ collisions for $p_{\mathrm{T}}>2-3 \mathrm{GeV}$ but over-predicts soft hadrons due to its power law divergence. We use the the results of the soft string models that fit $p p$ and $\bar{p} p$ data to parametrize by a simple exponential $p_{\mathrm{T}}$ behavior (in the $m \rightarrow 0$ limit) the low $p_{\mathrm{T}}$ region. If similar soft string dynamics drives $p p, p A$ and $A A$ collisions then most of the global observables with the exception of the baryon-antibaryon asymmetry can also be inferred from already existing data. The FNAL E-735 data [15] on hadronic spectra in $\bar{p} p$ at $\sqrt{s}=200 \mathrm{GeV}$ suggests values for the particle ratios that come very close to preliminary RHIC data (shown for comparison in parentheses). The $\bar{p} / \pi=0.07(0.08)$. For $K / \pi=0.11(0.15)$ we find $\sim 30 \%$ deviation. For pions and kaons one infers from charge and strangeness conservation that $\pi^{+} / \pi^{-}=1.0(1.0)$ and $K^{+} / K^{-}=1.0(1.1)$. The ratio that cannot be fixed from data on net baryon-free $\bar{p} p$ collisions is $\bar{p} / p$. However, it can be predicted by baryon transport (Regge) theory, i.e. $d N^{B} / d y=Z \beta \cosh \left(\left(1-\alpha_{B}(0)\right) y\right) / \sinh \left(\left(1-\alpha_{B}(0)\right) Y_{\max }\right)$ with a coupling $\beta=\mathcal{O}(1)$. In the baryon Junction picture $\alpha_{B}(0) \simeq 1 / 2$ [11]. For $A u+A u$ collisions at RHIC at $\sqrt{s}=130 \mathrm{AGeV}\left(Y_{\max }=5.4\right)$, the prediction $d N^{B} / d y \sim d N^{p} / d y-d N^{\bar{p}} / d y \simeq 11$ is close to the preliminary data. The predicted Junction trajectory slope, $\alpha_{J}^{\prime} \approx \alpha_{R}^{\prime} / 3$, implies that the effective string tension $\kappa^{\prime}$ is three times higher than $\kappa=1 /\left(2 \pi \alpha_{R}^{\prime}\right) \approx 1 \mathrm{GeV} / \mathrm{fm}$. This naturally leads to $\left\langle p_{\mathrm{T}}^{2}\right\rangle_{J} \approx 3\left\langle p_{\mathrm{T}}^{2}\right\rangle_{R}$, i.e. $T_{0}^{p} \simeq \sqrt{3} T_{0}^{\pi}$. Given the pion mean inverse slope, $T_{0}^{\pi} \simeq 220 \mathrm{MeV}$, it follows that the proton and antiproton slopes should be $T_{0}^{p} \simeq 400 \mathrm{MeV}$ for the junction (high $p_{\mathrm{T}}$ ) component of those yields. 

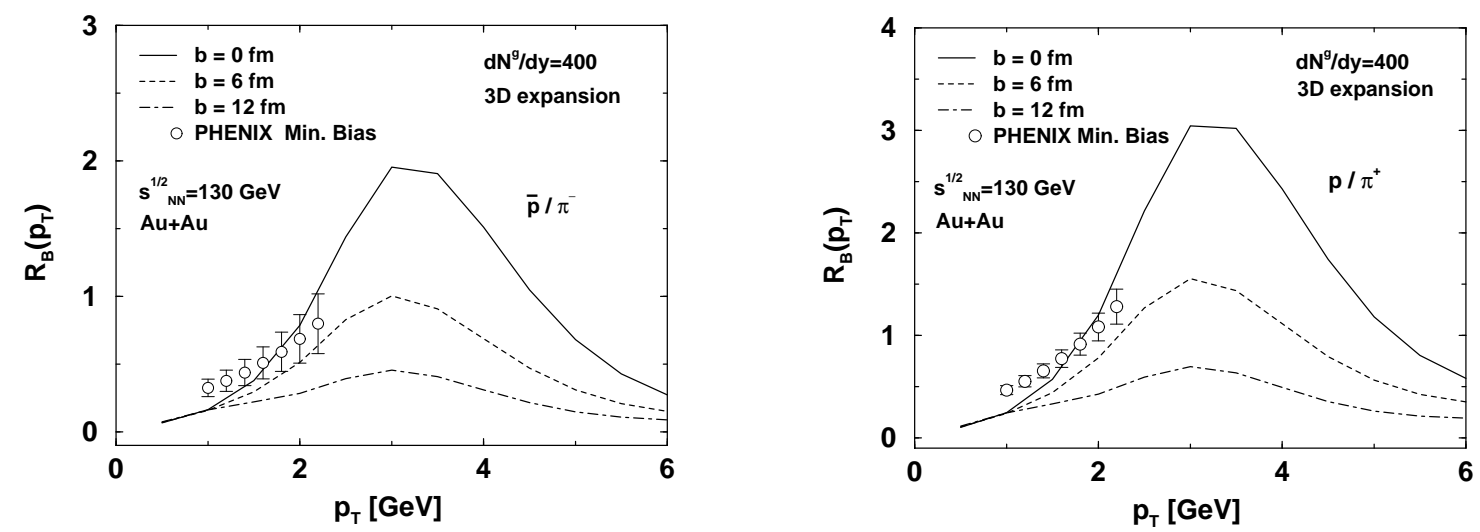

FIGURE 2. The $\bar{p} / \pi^{-}$and $p / \pi^{+}$ratios are shown in Figs. $2 \mathrm{a}, 2 \mathrm{~b}$ as a function of $p_{\mathrm{T}}$ for 3 different impact parameters $\mathbf{b}=0,6,12 \mathrm{fm}$. The ratio of out fits to preliminary minimum bias PHENIX data is shown for comparison.

Published RHIC data [4] constrain the charged particle multiplicity at midrapidity to $d N^{h} / d y(\mathbf{b}=0) \simeq$ 600. Thus with one parameter, the pion mean inverse slope (the $T_{0}^{K}=275 \mathrm{MeV}$ is irrelevant for the study of the $\bar{p} / \pi^{-}$and $p / \pi^{+}$ratios), the above theoretical analysis based on the string and baryon Junctions picture results in a solvable set of equations for the parameters in the phenomenological soft component of the hadronic spectrum:

$$
\frac{d N_{s}^{h}(\mathbf{b})}{d y d^{2} \mathbf{p}_{\mathrm{T}}}=\sum_{\alpha=\pi, K, p, \cdots} \frac{d n^{\alpha}(\mathbf{b})}{d y} \times \frac{e^{-p_{\mathrm{T}} / T_{0}^{\alpha}(\mathbf{b})}}{2 \pi T_{0}^{\alpha 2}(\mathbf{b})} .
$$

This component is assumed to scale with the number of participants, although the Junction component may scale faster [11]. Eqs. (11,3) give the hadron inclusive multiplicity

$$
\frac{d N^{h}(\mathbf{b})}{d y d^{2} \mathbf{p}_{\mathrm{T}}}=\frac{d N_{s}^{h}(\mathbf{b})}{d y d^{2} \mathbf{p}_{\mathrm{T}}}+T_{A A}(\mathbf{b}) \frac{d \sigma^{p p}}{d y d^{2} \mathbf{p}_{\mathrm{T}}}
$$

where $T_{A A}(\mathbf{b})$ is the Glauber profile density per unit area. The power law PQCD component is limited to the range $p_{\mathrm{T}}>2 \mathrm{GeV}$. If the Junction and Junction loop components scales nonlinearly with $N_{\text {part }}(b)$, as in HIJING / $B \bar{B}$, then the rate of change of the maximum of $R_{B}$ with increasing $\mathbf{b}$ would change correspondingly.

\section{CONCLUSIONS}

Fig. 1b shows the ratio of the charged particle multiplicities computed in our model relative to unquenched scaled PQCD (which fits to the UA1 data [16]). The high $p_{\mathrm{T}}$ tails show approximately constant suppression driven by the initial gluon density. Similar computation is shown for neutral pions for mean energy loss driven calculation with $d N^{g} / d y=400$. In the intermediate $p_{\mathrm{T}}=2-5 \mathrm{GeV}$ region the factor of 2 difference in the apparent suppression arises from the anomalously large baryon and antibaryon component predicted by the Junction picture.

In going from central to peripheral collisions the smaller partonic densities result in larger typical sizes of strings and Junctions and somewhat smaller mean inverse slopes of the resulting soft hadrons. This effect is taken into account by introducing a difference $\Delta T_{0}^{\pi}=-10 \mathrm{MeV}, \Delta T_{0}^{K}=-25 \mathrm{MeV}$ and $\Delta T_{0}^{p}=-50 \mathrm{MeV}$ between central $\mathbf{b}=0 \mathrm{fm}$ and peripheral $\mathbf{b}=12 \mathrm{fm}$ collisions. The major distinction between various centralities, however, is the different strength of jet quenching due to the different lengths of the path that the jet travels inside the plasma and the different density of the medium. Thus in peripheral collisions the baryon/meson ratio is expected to stay below unity similar to the $\bar{p} p$ case. Figs. 2a and $2 \mathrm{~b}$ show the hybrid two component model predictions for the $\bar{p} / \pi^{-}$and $p / \pi^{+}$ratios as a function of transverse momentum and 
centrality. While the precise values $R_{B}$ max of the maximum baryon and antibaryon excess are sensitive to the uncertainties in the estimates of the plasma density and the mean inverse slopes, the characteristic $p_{\mathrm{T}}$ and $\mathbf{b}$ behavior is not. In Figs. $2 \mathrm{a}$ and $2 \mathrm{~b}$ we have included the ratio of our exponential fits in a narrow $p_{\mathrm{T}}=1-2.1 \mathrm{GeV}$ window to the preliminary PHENIX minimum bias data [10]. The error bars reflect the one $\sigma$ uncertainties in the slope and intercept parameters.

Unlike hydrodynamics the dual soft + hard hybrid model predicts that the baryon and antibaryon anomaly is in fact limited to finite $p$ window and the baryon to meson/ratios eventually converge to the PQCD dominated base at high $p_{\mathrm{T}}$. It also predicts the systematic decrease of the predicted ratios with impact parameter which can be tested at RHIC. With higher statistics the dynamics of the baryon/meson ratios as a function of centrality may fact be probed in the current experimentally accessible $p_{\mathrm{T}}$ range.

\section{REFERENCES}

1. Quark Matter 2001, http://www.rhic.bnl.gov/qm2001/program.html

2. David, G, [PHENIX Collaboration], nucl-ex/0105014; Zajc, W.A., [PHENIX Collaboration], nucl-ex/0106001.

3. Ackermann, K.H. et al., [STAR Collaboration], Phys. Rev. Lett. 86, 402 (2001), nucl-ex/0009011; Snellings, R.J., [STAR Collaboration], nucl-ex/0104006.

4. Back, B.B. et al., [PHOBOS Collaboration], Phys. Rev. Lett. 85, 3100 (2000), hep-ex/0007036]; Adcox, K. et al., [PHENIX Collaboration], Phys. Rev. Lett. 86, 3500 (2001), nucl-ex/0012008].

5. Wang, X.-N. and Gyulassy, M., Phys. Rev. Lett. 86, 3496 (2001), nucl-th/0008014.

6. Gyulassy, M., Vitev, I and Wang, X.-N., Phys. Rev. Lett. 86, 2537 (2001); Lévai, P. et al., nucl-th/0104035.

7. Gyulassy, M., Lévai, P., Vitev, I., Phys. Rev. Lett. 85, 5535, (2000); Nucl. Phys. B594, 371 (2001).

8. Aggarwal, M.M. et al., [WA98 Collaboration], Phys. Rev. Lett. 81, 4087 (1998); Erratum-ibid. 84, 578 (2000).

9. Wang, X.-N., Phys. Rev. Lett. 81, 2655 (1998), hep-ph/9804384.

10. Velkovska, J., [PHENIX Collaboration], nucl-ex/0105012.

11. Rossi, G.C. and Veneziano, G., Nucl. Phys. B123, 507 (1977); Kharzeev, D., Phys. Lett. B378, 238 (1996); Kopeliovich, B.Z. and Zakharov, B.G., Z. Phys. C43, 241 (1989); Phys. Lett. B381, 325 (1996); Vance, S.E., Gyulassy, M. and Wang, X.-N., Phys. Lett. B443, 45 (1998); Vance, S.E. and Gyulassy, M., Phys. Rev. Lett. 83, 1735 (1999).

12. Xu, N. and Kaneta, M., [STAR Collaboration], nucl-ex/0104021; Harris, J., "Results from STAR", http://www.rhic.bnl.gov/qm2001/program.htm

13. Vitev, I. and Gyulassy, M., nucl-th/0104066; Vitev I. and Gyulassy M., work in progress.

14. Gyulassy, M., Levai, P. and Vitev, I., in preparation.

15. Alexopoulos, T. et al., [E735 Collaboration], Phys. Rev. D48 984 (1993).

16. Albajar, C et al, [UA1 Collaboration], Nucl. Phys. B335, 261 (1990). 